\title{
Eine neue Talmudübersetzung.
}

\section{Eine Warnung.}

Von A. Sulabach in Frankfurt a. M.

-Eine Übersetzung des babylonischen Talmud ${ }^{1}$, wenn sie wahrheitsgetreu und weiten Kreisen verständlich ist, wird man von allen Seiten ohne Ausnahme begrüßen. Sie wird Klarheit bringen und häßliche Zwistigkeiten und Anfeindungen aus der Welt schaffen, sie wird kulturhistorische Werte aufdecken und der Wissenschaft im allgemeinen dienen, sie wird im besonderen auch für die Kirchengeschichte und die Forschung in der neutestamentlichen Literatur von unschätzbarem Werte sein, da die Bedeutung des Talmud für die Erkenntnis vieler Stellen im NT, wie auch vice versa von den Männern der Wissenschaft längst anerkannt ist.

Natürlich muß derjenige, der sich an die Riesenaufgabe der Übersetzung des Talmud heranwagt, diesen so kennen, daß er weiß, er stehe vor einer Riesenaufgabe; vor allem aber muß der Übersetzer ein großes $\mathrm{MaB}$ von Kenntnis und Wissenschaft mitbringen, er muß den Talmud sprachlich und inhaltlich verstehen, etwas, das man ja von jedem Übersetzer eines fremdsprachlichen Werkes erwartet, wenn der Wert seiner Arbeit den des Papiers übersteigen soll, das für die Niederschrift verwendet worden ist. Man sollte meinen, dies alles sei eine Binsenwahrheit, die nicht erst erörtert werden müßte, jedoch aus dem Folgenden wird man erkennen, daß dies nicht für alle eine ausgemachte Sache ist, daß selbst ein Professor über diese Voraussetzungen, in Selbsttäuschung befangen, sich nicht klar ist und sich an Arbeiten wagt, die er nicht bemeistern kann.

Herr Schlögl hat sich nun, da die bisherigen Leistungen auf dem Übersetzungsgebiet des Talmud mangelhaft, fehlerhaft, also unbefriedigend seien, entschlossen, wie der Verlag mitteilt, »eine streng wissenschaftliche, richtig und gut deutsche Übertragung des Talmud zu bringen, die weiten Kreisen zugänglich und verständlich ist". Alle sechs Wochen soll eịne Lieferung erscheinen; wenn nun der ganze Talmud erscheinen soll, so wird in wenigen Jahren die Arbeit fertig sein, und die Welt wird einen Rekord von Schnelligkeit erleben, der bisher gewiß nicht erreicht worden ist.

1 Der babylonische Taimud. Übersetzt und kurz erläutert von Dr. Nivard Schlögl, O. Cist., 0. o. Professor für orientalische Sprachen a. d. Wiener Univers. x. Lieferung. $96 \mathrm{~S}$. Wien r921, Burgverlag Richter und Zöllner. 
Ist nun Herr Schlögl der Mann, der uns für eine getreue, allgemeinverständliche Übersetzung des Talmud noch fehlt? Ein dürres Heftchen von nur 96 Seiten, noch nicht der zehnte Teil einer vollendeten Übersetzung, liegt vor, und zwar die Übersetzung des verhältnismäßig leichtesten Trakats, Berakoth, und doch ist es nicht leichtfertig, nicht allein das vorliegende Bruchstück $\mathrm{zu}$ beurteilen, sondern auch zu erklären, was die Welt von den Fortsetzungen noch zu erwarten hat.

Die vorliegende Probe ergibt, daß alles, was wir von einem Übersetzer erwarten dürfen, fehlt: Sachkenntnis und Wortkenntnis und dadurch auch die Fähigkeit, den Stoff dem Verständnis des Lesers nahezubringen.

$\mathrm{Um}$ mit dem Letzten $z \mathrm{u}$ beginnen, greife ich aus vielem folgenden Satz heraus: „Wenn einer es im Gesetze liest und es gerade Zeit ist, es aufzusagen (sic!) und er denkt daran, so ist er seiner Pflicht ledig* (S. 65). Nun möchte ich den kennen, der dieses Deutsch versteht, falls ihm dieser. Satz aus dem Originaltext nicht verständlich ist. Was ist »es«? Freilich wenn der Leser 22 Seiten zurückblättert, findet er es vielleicht. Ferner wer denkt daran«, woran? Ja, wenn der Verf. selbst wüßte, was kiwan libo bedeutet, dann hätte er wohl wortrichtiger, aber nicht verständlicher übersetzt. Dies eine Beispiel für viele.

Wie steht es aber nun mit dem allgemein talmudischen Wissen des Verf.? Es ist doch eigentümlich, daß der Mann, der sich als den Übersetzer schätzt, in. den zwei ersten Zeilen (sage zwei ersten Zeilen!) seines.Werkes sich eine Bloße gibt, seine Unwissenheit dokumentiert, wie man sie einem Anfänger im Talmudstudium nicht verzeihen würde. Er übersetzt נכנם"ן (nichnassin) mit "sich versammeln * und Terumah mit Hebe, wozu die Anmerkung »Opferanteil, vgl. Lev. 22, 4-7.* So viel Worte, so viele Fehler. עכנם heißt, wie allbekannt, "hineingehen «, nicht »versammeln «, das wäre die Bedeutung im Hitpael, und Teruma ist kein Opferanteil, der nur in Jerusalem verzehrt werden dürfte, bzw. im Tempelrevier, hier ist aber vom ganzen Lande die Rede, und es wird gesagt: die Zeit des Schemálesens am Abend beginnt zu der Zeit, da die Priester, die levitisch unrein waren und das Tauchbad vor der Stadt genommen haben, hineingehen (sc. in die Stadt), um in ihren Häusern die Teruma (Naturalabgaben als Entschädigung für ihren Ausschluß am Landbesitz) nach Sonnenuntergang in Reinheit $z u$ verzehren. Das alles weiß Herr Schlogl nicht und so verdreht er den Sinn des Satzes und führt die Leser irre. Das ist aber wahrhaftig eine gute Empfehlung für ein Werk, 
wenn es an seiner Spitze schon ein so hübsches Gesicht zeigt. Ich meine, das sollte eigentlich für die Urteilsbildung über die Kapazität des Verf. und somit auch über das, was wir von der Fortsetzung seiner Arbeit erwarten dürfen, genügen, allein nicht das eine soll maßgebend sein, ich will aus der Fülle von Fèlern, die uns hier begëgnen, noch einige wenige heraussuchen, um mir nicht den Vorwurf ungerechten Urteilens zuzuziehen.

S. 9: „Man rede in Gegenwart eines Toten nur Worte des Toten (Ber. 3 b). Der Verf. weiß nicht, daß רבר nicht nur Wort, sondern auch Angelegenheit bedeutet, der Sinn also ist, aus Achtung vor der Leiche spreche man in ihrer Gegenwart nicht unnützes Gerede, sondern nur das, was die Leiche betrifft: Waschung, Einkleidung, Bestattung usw. Das will der Talmud sagen und nicht den Unsinn, den der Verf. ihm unterschiebt. Ad vocem רב sei hier gleich auf S. 39 hingewiesen, wo dem Verf. ein ähnliches Unglück passiert ist. Den Persern wird nachgerühmt, sie seien unter anderem צנועטין (eigentlich bescheiden, zurückgezogen) in דבר אחר, was (Ber. 8b) geschmackvoll mit »keusch auch sonst" übersetzt ist; dies hat ihm das דבר angetan, er weiß nämlich nicht, daß דו"או

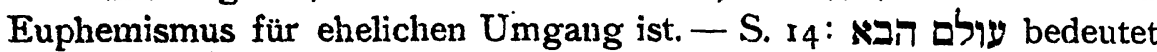
im Talmud, sobald es für das Dasein nach dem Tode angewandt wird, nur die Seligkeit und nicht auch die Hölle. - S. 23: (das. 6a) bedeutet nicht Alleinherrscher, was dem Verf. schon das $i$ hätte sagen müssen, sondern "Gegenstand der Erwählung" (vgl. Dalmann, Aram.-Neuhebr. Wtb.). - S. 43: "Bis zur dritten Stundex, dazu. im Notenparterre die richtige Anmerkung: "Neun Uhr vormittags «, der Text fährt nun aber merkwürdigerweise fort, »denn so ist es usw. Brauch um 3 Uhr aufzustehen «. Haben hier zwei Hände, die eine im Text, die andere richtig geführte in den Anmerkungen, gearbeitet? - Das. מצודה (Ber. gb) heiBt nicht »Netzk, sondern 》Festung* und וינצו nicht ssie beraubten" sondern »sie leerten ausк. - Den Gipfel der Lächerlichkeit hat der Verf. erstiegen, wenn er S. 44 Maimonides unterschiebt, er habe unter בין תכלת ללבן (Ber. gb) verstanden, die Zeit für das Morgenschemá beginne, wenn es so hell jst, daß man an dem weißen Gebetmantel die blauen Streifen erkennen könne. Zu Zeiten der Mischna der weiße Gebetmantel und zur Zeit des Maimonides die späterer Mode angehörenden blauen Streifen! Das ist kostbar. Maimonides spricht von den Schnufäden, Zigith, und der Verf. unterschiebt ihm das. Talith, und M. sagt nichts anderes, als die Helle, die genügt, um im Purpurfaden an den Schnu- 
fäden die etwa noch vorhandenen weißen, nicht hinreichend gefärbten Stellen zu erkennen, genüge, um das Tagesschemá sagen zu dürfen. Was ich hier an Beweisen für die.Unfähigkeit des Herrn Schlögl, eine sinn- und wahrheitsgetreue Übersetzung des Talmud zu liefern, dem Leser vor Augen gestellt habe, ist nur ein kleiner Bruchteil von dem, was meine Prüfung an Schnitzern und allem, was unter diesen zu rangieren ist: Unkenntnis der Sprache, der Terminologie, des Geistes des Talmud usw., mir ergeben hat. Doch ich denke, es ist genug, und möchte mich mit einigen Worten über das Parterre der Anmerkungen aussprechen, für die Herr Schlögl, da sie unter seiner Flagge segeln, die Verantwortung hat, obwohl ich, wie ich schon oben angedeutet, gute Gründe habe, sie für die Arbeit einer $z$ weiten Hand zu halten.

Die Anmerkungen bestehen zum Teil in moquanten Glossen zu den Aussprüchen, Deutungen und Anschauungen der Rabbinen. Nun mag ja der Verf. sich moquieren, soviel er will, das kann ihm keiner wehren; aber nur als Kritiker, nicht als Übersetzer. Dem Übersetzer muß das Werk, das er übersetzt, ganz objektiv gegenüberstehen; ob die Handlungen oder Meinungen ihm gefallen oder nicht, das hat er nicht kundzugeben, er diskrediert das Werk, dem er sich widmet, in dem Moment, wenn der Leser es in die Hand nimmit. $\mathrm{Ei}$, wenn es so schlechtes Zeug ist, warum hat er sich für die Weiterverbreitung so bemüht? - - Ferner liefert der Verf. den Lokalnachweis für die angeführten Bibelzitate, dazwischen fallen aber die Bemerkungen: Einschub, verdorbener Text, messianisch usiv. Ja, was soll denn das? Soll den Rabbinen etwa ein Vorwurf gemacht werden, daß sie nach dem ihnen vorgelegenen Text, der ja überdies heute noch der offizielle ist, und nicht wach den Ergebnissen der Bibelkritik der Neuzeit zitieren? Oder verlangt er, daß die Rabbinen die Auslegungen der Kirche sich hätten aneignen sollen? Antwort: Tendenz, wie sie stellenweise auch aus dem Text hervorlugt; mit Tendenz aber schafft man kein wissenschaftliches Werk.

Schließlich, Herr Schlögl ist nicht der Mann, eine der Wissenschaft dienende oder die Welt aufklärende Übersetzung des Talmud herzustellen. Er kann nur eine Übersetzung des Talmud geben, die diesen entstellt. Manchem mag ja eine solche Karikatur des Talmud erwünscht sein, aber diese kann der Wissenschaft nichts nützen, die Welt nicht aufklären, sie kann nur Unheil anrichten.

[Abgeschlossen am 1. Februar 1922.] 